\title{
Effect of Filler on Glass Transition of Asphalt Mastics
}

\author{
Meng Guo ${ }^{1, a}$, Yi-qiu Tan ${ }^{2, b}$ and Lei Zhang ${ }^{3, c}$ \\ ${ }^{1}$ School of Transportation Science and Engineering, Harbin Institute of Technology, Harbin 150090, \\ Heilongjiang, PR China \\ ${ }^{2}$ School of Transportation Science and Engineering, Harbin Institute of Technology, Harbin 150090, \\ Heilongjiang, PR China \\ ${ }^{3}$ School of Transportation Science and Engineering, Harbin Institute of Technology, Harbin 150090, \\ Heilongjiang, PR China \\ aguomeng87@163.com, byiqiutan@163.com, chit.andy@foxmail.com
}

\begin{abstract}
Keywords: Filler, Asphalt Mastics, Glass Transition, Dynamic Mechanic Analysis; Differential Scanning Calorimetry
\end{abstract}

\begin{abstract}
A study has been carried out on the glass transition of fifteen asphalt-filler mastics in three different filler types. The dynamic mechanical analysis (DMA) and differential scanning calorimetry (DSC) method were used. The results show that DMA method can measure the glass transition temperature (Tg) of asphalt-filler mastics more accurately than DSC; The glass transition temperature measured by DMA is generally higher by $20 \sim 40^{\circ} \mathrm{C}$ than that measured by DSC; The glass transition temperature of the asphalt-filler mastics increase with the increase of the filler volume fraction, and andesite is the most sensitive; When the volume fraction of filler is greater than 0.5 , the enhance capability of glass transition temperature of asphalt-filler mastics is in the order of andesite $>$ granite $>$ limestone, when the volume fraction of filler is smaller than 0.2 , the enhance capability is in the order of granite $>$ limestone $>$ andesite.
\end{abstract}

\section{Introduction}

Asphalt-filler mastics is an important part of asphalt mixture, and its properties influences the performance of asphalt pavement directly. Fillers are used to lower the cost of materials, increase rigidity and give special properties to a material (such as colour or fire retardancy). The filler volume fraction and properties have considerable effects on the processing characteristics of materials such as mixing, pumping and compacting. The effects of fillers are therefore of vital importance. The performance of asphalt-filler mastics is different from base asphalt and asphalt mixtures.

Physical and rheological tests are known to correlate well with road performance. A certain similarity exists between the viscoelastic behavior of simple amorphous polymers and asphalt [1]. As the glass transition temperature ( $\mathrm{Tg}$ ) is a very important parameter of amorphous polymers [2], it should also be useful in interpreting the properties of asphalts. It is a reversible change in an amorphous domain from a viscous or rubbery state to a hard and relatively brittle glassy state, and vice versa.

Thermal analysis and dynamic mechanical analysis provide the possibility of determining $\mathrm{Tg}$ and morphology. Y. Edwards [3] used dynamic mechanical analysis (DMA) and differential scanning calorimetry (DSC) to demonstrate that bitumen composition was of decisive importance, and adding polyethylene wax or polyph-osphoric acid especially to a non-waxy bitumen, showed considerable positive effects on the rheological behaviour at medium and higher temperatures. P. Starck [4] measured the elastic modulus (stiffness), the glass transition temperature (Tg) of the bitumen phase, and the softening temperature by using DMA, and demonstrated that the viscosities of the blends increased significantly after the immersion in de-icing agents. Kim YR [5] used DMA to evaluate the fatigue and healing potential of asphalt binders in sand asphalt mixtures, and he considered several candidate fatigue failure parameters to assess fatigue life and the effect of rest periods on the extension of fatigue life through healing. Pavel Kriz [6] applied DSC to asphalt binders and 
successfully identified binders that are sensitive to time hardening at low temperatures. In such binders, several thermodynamic and morphology parameters are strongly time-dependent, suggesting that the viscoelastic properties change with time. Wu SP [7] studied the thermal behavior and characterization of the ultraviolet aged asphalt binder. Their results indicated that the UV light ageing would lead to the improvement of thermal behavior and the growth of the glass transition temperature of asphalt binder. M. Garcla-Morales [8] conclude that the viscous properties of bitumen, at high temperature, are improved by adding recycled EVA copolymer in amounts that depend on bitumen penetration grade. Moreover, significant microstructural changes, related to the development of a polymer-rich phase, tend to occur in the bitumen as polymer concentration increased.

\section{Materials and test methods}

2.1. Experimental materials. Experimental materials used in this paper include $90 \#$ base asphalt and three kinds of mineral fillers, and their technical data are shown in Table 1 and Table 2. Fifteen asphalt mastics are prepared by mixing asphalt and fillers for 15 minutes at speed of $1000 \mathrm{rpm}$. Asphalt mastics are divided into three categories (limestone asphalt mastic, andesites asphalt mastic and granite asphalt mastic), and each categories include five filler volume fraction $(\Phi=0.1,0.2,0.3$, 0.4 and 0.5 ).

Table 1. Technical data of $90 \#$ base asphalt

\begin{tabular}{cc}
\hline Test item & Measured value \\
\hline $25^{\circ} \mathrm{C}$ Penetration $(0.1 \mathrm{~mm})$ & 83.0 \\
Softening point $\left({ }^{\circ} \mathrm{C}\right)$ & 46.3 \\
$15^{\circ} \mathrm{C}$ Ductility $(\mathrm{cm})$ & $>140$ \\
\hline
\end{tabular}

Table 2. Physical properties of mineral powder

\begin{tabular}{ccccc}
\hline Filler type & Density $\left(\mathrm{g} / \mathrm{cm}^{3}\right)$ & \multicolumn{3}{c}{ Sieve percentage $(\%)$} \\
\cline { 3 - 5 } & & $0.6 \mathrm{~mm}$ & $0.15 \mathrm{~mm}$ & $0.075 \mathrm{~mm}$ \\
\hline Limestone & 2.827 & 100 & 96.5 & 76.7 \\
Andesites & 2.864 & 100 & 98.7 & 76.7 \\
Granite & 2.614 & 100 & 85.7 & 74.2 \\
\hline
\end{tabular}

2.2. Laboratory testing. Differential scanning calorimeter used in this paper is DSC200F3 produced by NETZSCH GmbH. The temperature range of tests is from $-100^{\circ} \mathrm{C}$ to $+100^{\circ} \mathrm{C}$. Glass transition temperature is determined by analyzing the curve showing the relationship between heat flow and temperature (Figure 1). Pavel Kriz [6] pointed out that the repeatability and accuracy of Tg inflection point is good, but multi-component material include many Tg inflection point like asphalt, so using only one Tg inflection point can't show the whole property of the material; Tg midpoint can reflect the average of the glass transition region, but the value of Tg midpoint are sensitive to the selection of the start point and the end point, so this paper analyzed both of them. At first, we studied the influence of heating rate on measurement results (Figure 3 ). 


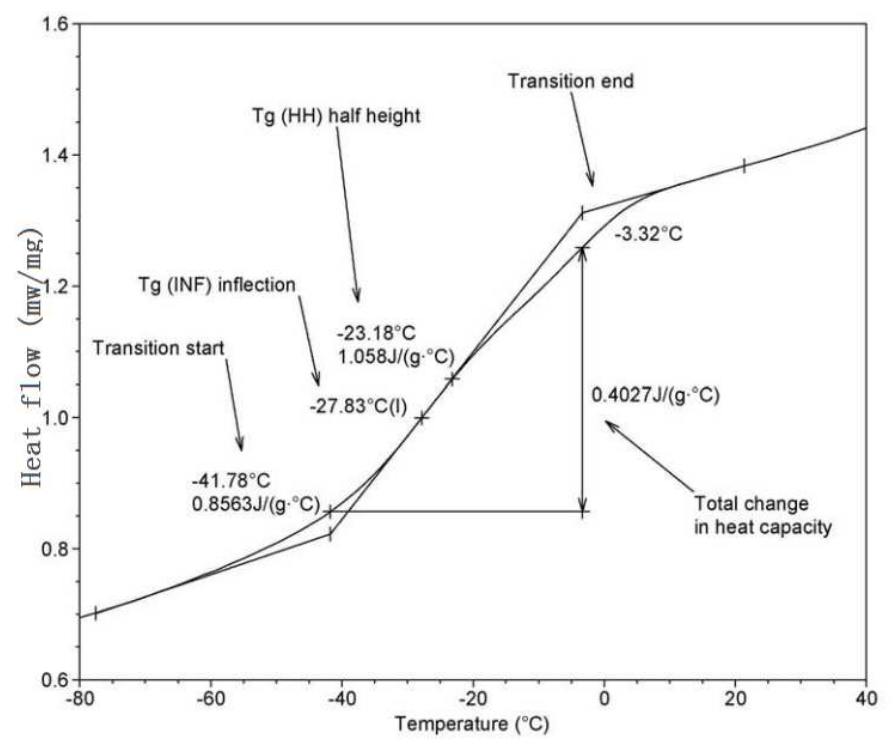

Fig.1. Determination of glass transition temperature by DSC.

Dynamic mechanical analysis method is an effective means of study the changing of polymer structural, molecular motion and performance. In this paper, the dynamic mechanical thermal analyzer DMA Q800 produced by TA was used to do a dynamic tension and compression testing on the asphalt-filler mastics, and the test parameters were selected as follows:

Temperature range: $-60^{\circ} \mathrm{C} \sim+50^{\circ} \mathrm{C}$;

Heating rate: $5^{\circ} \mathrm{C} / \mathrm{min}$;

Initial isothermal conditioning: 3 minutes;

Load mode: strain control, amplitude $=0.05 \%$, frenquecy $=1 \mathrm{~Hz}$;

Specimen size: $17.33 \mathrm{~mm} \times 4.50 \mathrm{~mm} \times 2.40 \mathrm{~mm}$.

We can get the relationship of storage modulus, loss modulus, phase angle and temperature by doing the above test (Figure 2). By analyzing Figure 2, we can see that the peak value of loss modulus can reflect the glass transition temperature of asphalt-filler mastics accurately, but the cross point of tangents of storage modulus is imprecise in determining Tg of asphalt-filler mastics, because it's sensitive to the selection of the start point and the end point.

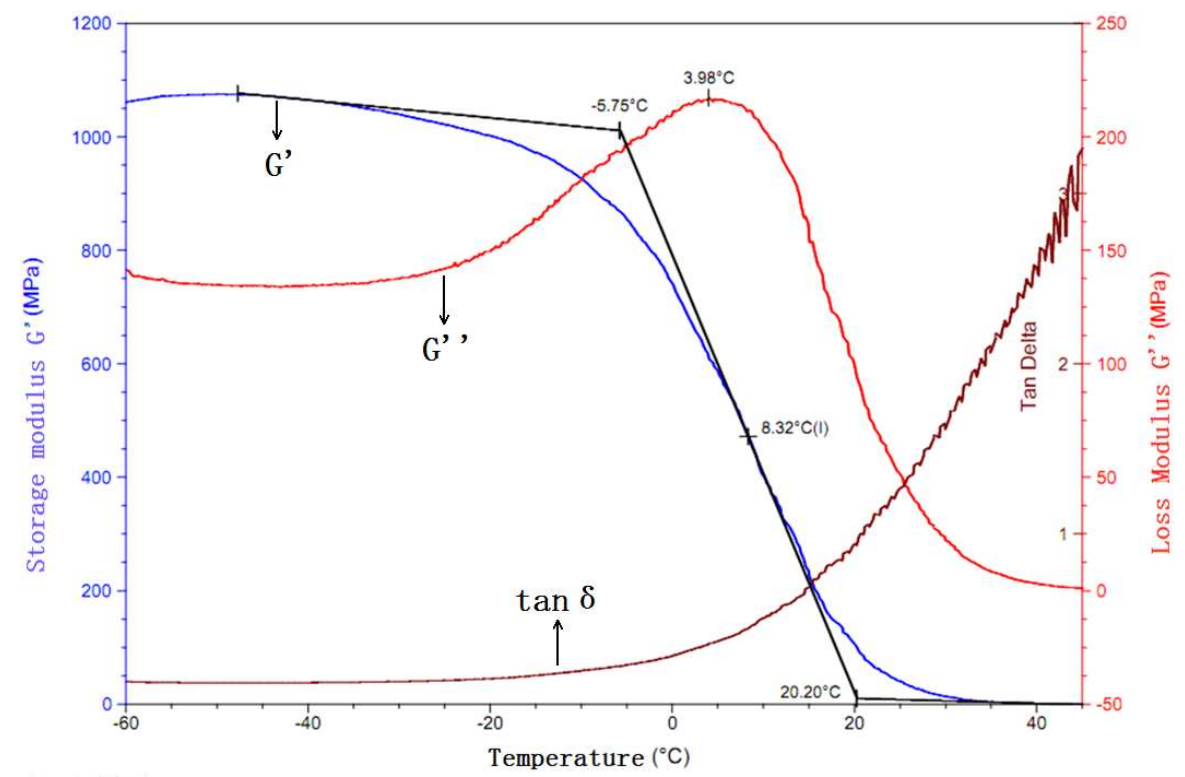

Fig.2. Determination of glass transition temperature by DMA. 


\section{Results and discussion}

\subsection{Results of DSC tests.}

(1) The influence of heating rate on glass transition

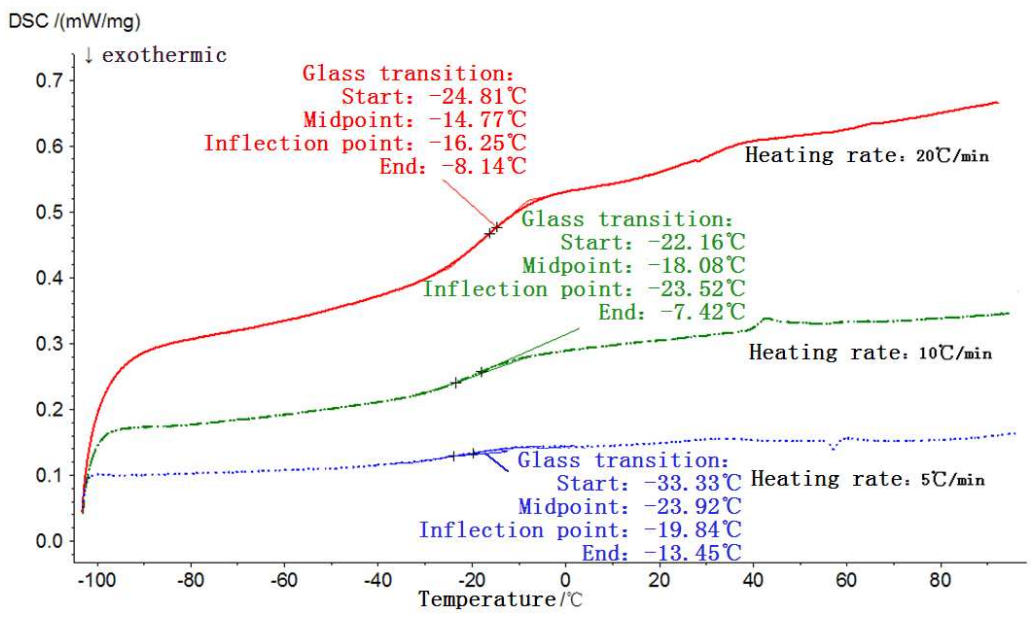

Fig.3. The influence of heating rate on glass transition.

It can be seen from Figure 3, with increasing heating rate, the glass transition is more obvious and more hysteretic, which is consistent with the time dependence of the chain segmental motion. If the time chain segment resisted stress is longer, they will have more time to make a response to the stress, the temperature of the glass transition will be much lower, which means that the glass transition is a dynamic process; The Tg midpoint increases with heating rate increases, but the Tg inflection point temperature presents ruleless.

(2) Effect of filler on Tg of asphalt mastic by DSC

The heating rate is set as $10^{\circ} \mathrm{C} / \mathrm{min}$, and the Tg measuring results of the various asphalt-filler mastics are shown in Figure 4 and Figure 5.

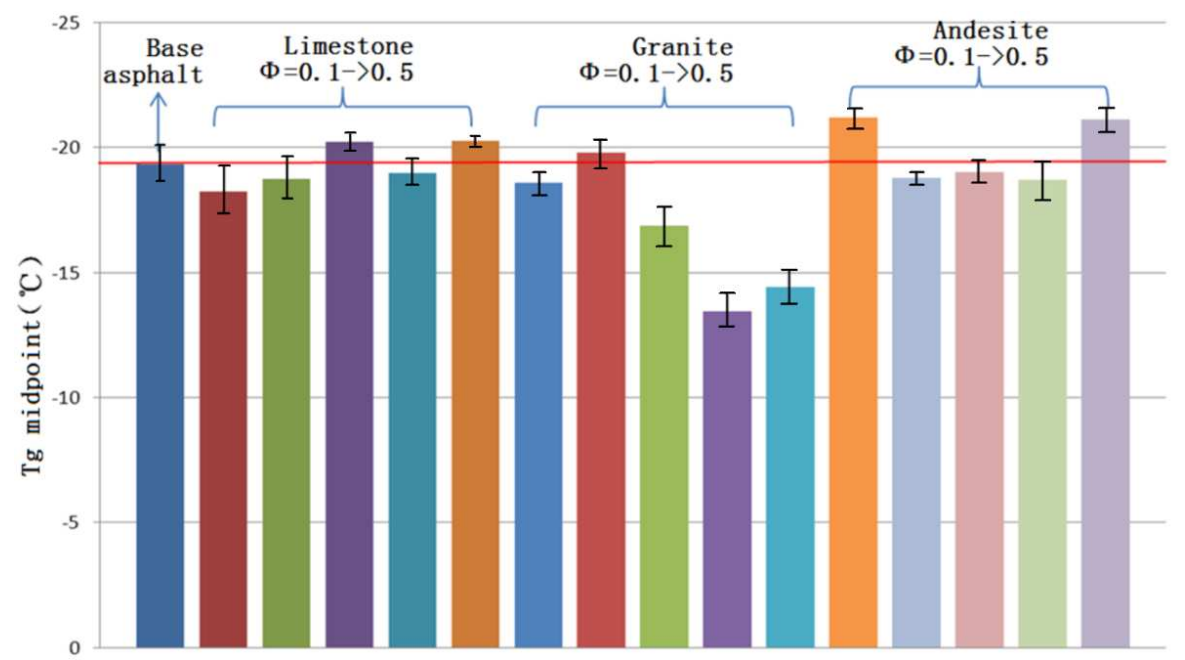

Fig.4. The Tg midpoint of various asphalt-filler mastics by DSC. 


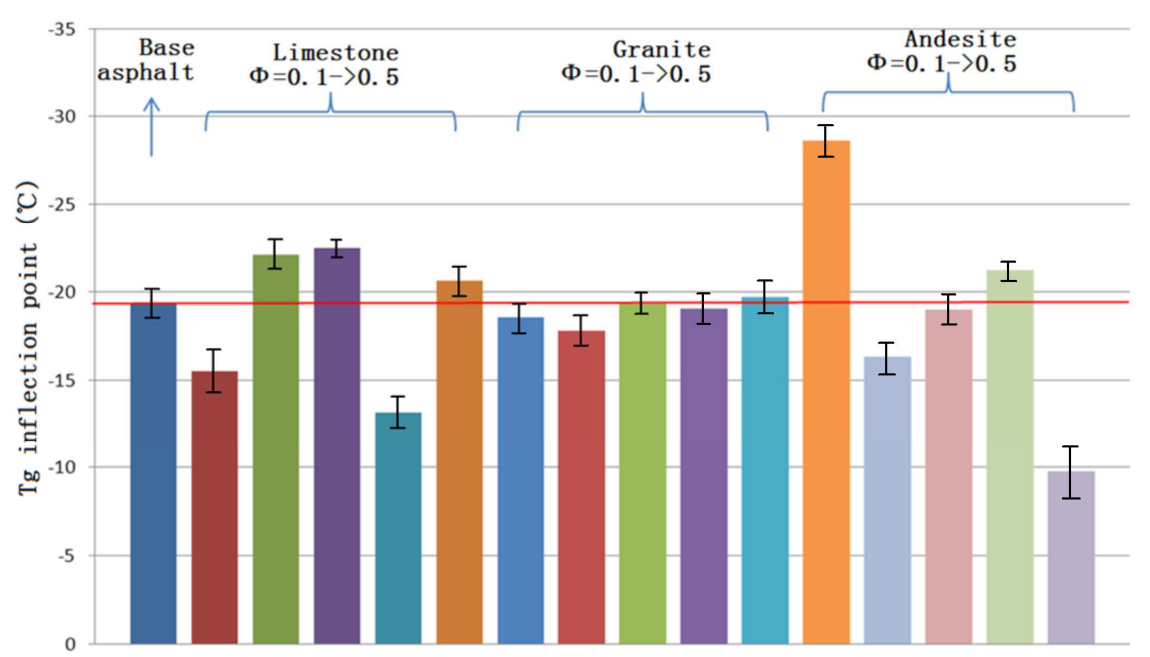

Fig.5. The Tg inflection of various asphalt-filler mastics by DSC.

It can be seen from Figure 4 and Figure 5 that Tg midpoint and Tg inflection point can't distinguish the glass transition of different asphalt-filler mastics clearly. The Tg midpoint of asphalt-filler mastics with granite filler has a weak regularity, which is the Tg midpoint increases with the increase of volume fraction.

\subsection{Results of DMA tests}

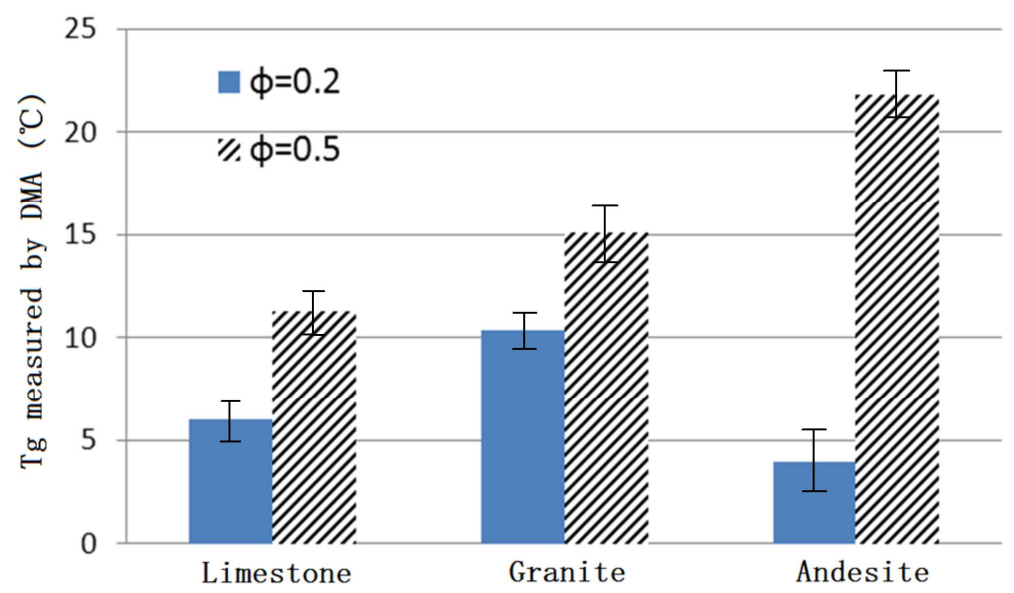

Fig.6. The Tg of various asphalt-filler mastics by DMA.

It can be seen from Figure 6 that dynamic mechanical analysis method has a better regularity in determining the glass transition temperature. The glass transition temperature of all asphalt-filler mastics have a same regularity, which is Tg increases with the increase of the filler volume fraction, and andesite is the most sensitive, which indicating that the more filler makes more interfacial layers, making the motion of asphalt molecular chain adsorbed on the surface of the filler particles become more difficult. The greater the filler content is, the stronger the effect is. When the volume fraction of filler is greater than 0.5 , the enhance capability of glass transition temperature of asphalt-filler mastics is in the order of andesite $>$ granite $>$ limestone, which means that the more andesite filler will make the worst low-temperature performance; while the volume fraction of filler is smaller than 0.2 , the enhance capability is in the order of granite $>$ limestone $>$ andesite, which indicates that less granite will the most decrease of glass transition temperature of asphalt-filler mastics.

By contrasting the Tg results obtained by the DSC method and DMA method, we can see that the DSC can't effectively reflect the influence of filling on the glass transition of the asphalt-filler mastics, while DMA have a considerable differentiation. The glass transition temperature measured by DMA is generally higher by $20 \sim 40^{\circ} \mathrm{C}$ than that measured by DSC, and the greater the filler volume fraction is, the greater the difference between the results of two methods is. 


\section{Summary and conclusions}

Based on the testing and analysis presented herein, the conclusions of the study are summarized as follows:

1) The DMA method can measure asphalt Tg of asphalt-filler mastics accurately, and it can evaluate the impact of the type and volume fraction of filler on $\mathrm{Tg}$. The glass transition temperature measured by DMA is generally higher by $20 \sim 40^{\circ} \mathrm{C}$ than that measured by DSC, and the more filler will make a bigger difference. The Tg midpoint measured by DSC has a weak regularity, and Tg inflection point has no regularity.

2) The DMA tests demonstrated that the glass transition temperature of the asphalt-filler mastics increase with the increase of the filler volume fraction, and andesite is the most sensitive.

3) When the volume fraction of filler is greater than 0.5 , the enhance capability of glass transition temperature of asphalt-filler mastics is in the order of andesite $>$ granite $>$ limestone; while the volume fraction of filler is smaller than 0.2 , the enhance capability is in the order of granite $>$ limestone $>$ andesite.

\section{References}

[1] Giavarini C, Pochetti F. Characterization of Petroleum Products by DSC Analysis. Journal of Thermal Analysis and Calorimetry, 5 (1973): 83-94.

[2] Ngai KL. The Glass Transition and the Glassy State, Physical Properties of Polymers, Cambridge University Press, Cambridge, UK, 2004: 72-152.

[3] Y. Edwards, Y. Tasdemir, U. Isacsson. Rheological effects of commercial waxes and polyphosphoric acid in bitumen 160/220 - high and medium temperature performance. Construction and Building Materials. 21 (2007): 1899-1908.

[4] P. Starck, B. LÖfgren. Influence of de-icing agents on the viscoelastic properties of asphalt mastics. J Mater Sci. 42(2007): 676-685.

[5] Kim YR, Little DN, Lytton RL. Use of dynamic mechanical analysis (DMA) to evaluate the fatigue and healing potential of asphalt binders in sand asphalt mixtures. Journal of the Association of Asphalt Paving Technologists. 71 (2002): 176-206.

[6] Pavel Kriz, Jiri Stastna, Ludo Zanzotto. Glass Transition and Phase Stability in Asphalt Binders. Road Materials and Pavements Design. 1 (2007): 1-30.

[7] Wu SP, Zhu GJ, Liu G, Pang L. Laboratory research on thermal behavior and characterization of the ultraviolet aged asphalt binder. Journal of Thermal Analysis and Calorimetry. 95 (2009): 595-599.

[8] M. Garcĺa-Morales, P. Partal, F.J. Navarroa, F. Martínez-Boza, C. Gallegos, N. González, O. González, M.E. Muñoz. Viscous properties and microstructure of recycled eva modified bitumen. Fuel. 83 (2004): 31-38. 